\title{
Relationships between Heart-Rate Variability and Pulse-Rate Variability obtained from video-PPG signal using ZCA
}

\author{
Luca Iozzia, Luca Cerina and Luca Mainardi \\ Department of Electronics, Information and Bioengineering, Politecnico di Milano, \\ via Golgi 39, 20133 Milano, Italy \\ E-mail: luca.iozzia@polimi.it
}

\begin{abstract}
.
In this paper, classical time- and frequency-domain variability indexes obtained by pulse rate variability (PRV) series extracted from video-photoplethysmography signal (vPPG) were compared with Heart Rate Variability (HRV) parameters extracted from ECG signals. The study focuses on the analysis of the changes observed during a restto-stand manoeuvre (a mild sympathetic stimulus) performed on 60 young, normal subjects (age: $24 \pm 3$ years). The objective is to evaluate if video-derived PRV indexes may replace HRV in the assessment of autonomic responses to external stimulation.

Video recordings were performed through a GigE Sony XCG-C30C camera and analyzed offline to extract the vPPG signal. A new method based on Zero-phase Component Analysis (ZCA) was employed in combination with a fully-automatic method for detection and tracking of Region of Interest (ROI) located on the forehead, the cheek and the nose.

Results show an overall agreement between time and frequency domain indexes computed on HRV and PRV series. However, some differences exist between rest and stand conditions. During rest, all the indexes computed on HRV and PRV series were not statistically significant different $(p>0.05)$ and showed high correlation (Pearson's $r>0.90$ ). The agreement decreases during stand especially for the high-frequency, respiration-related parameters such as RMSSD $(\mathrm{r}=0.75)$, pNN50 $(\mathrm{r}=0.68)$ and HF power $(\mathrm{r}=0.76)$. Finally, the power in the LF band (n.u.) was observed to significantly increase during stand by both HRV ( $28 \pm 14$ vs. $45 \pm 16$ (n.u.); rest vs. stand) and PRV (26 \pm 12 vs. $30 \pm 13$ (n.u.); rest vs. stand) analysis, but such an increase was lower in PRV parameters than that observed by HRV indexes.

These results evidence that some differences exist between variability indexes extracted from HRV and video-derived PRV, mainly in the HF band during stand. However, despite of these differences video-derived PRV indexes were able to evidence the autonomic responses expected by the sympathetic stimulation induced by rest-tostand maneuver.
\end{abstract}

\section{Introduction}

The measurement of cardiovascular parameters using contactless video-photoplethismography (vPPG) signal has gained larger and larger attention in the last decade [1-12]. 
Since the pioneer works by Poh and coworkers [3], a variety of solutions have been proposed to derive, from video of subject's face, physiological parameters such as heartrate [2] and breathing rate $[3,12]$ or to extract the ${ }^{\mathrm{PPPG}}$ signal [13], detect the pulse waves and to compute beat-to-beat variability parameters [5].

Recently, the agreement between pulse-rate variability (PRV) parameters (both in time and frequency domain) obtained from PPG and vPPG signals have been assessed in resting conditions in a limited group of subjects $[3,4,6]$. One paper employes PRV obtained by vPPG as a surrogate of HRV [11] during supine and sitting conditions. However, a still open question is whether PRV derived from vPPG can be considered a valid surrogate of the HRV parameters extracted from the RR interval series and whether they are able to describe changes in Autonomic Nervous System (ANS) modulation during provocative tests. To the extent of our knowledge, none of the study have addressed this issue systematically.

The purpose of this study is therefore to compare PRV parameters obtained from vPPG with HRV parameters computed on RR series and to evaluate the ability of vPPG parameters in detecting Autonomic Nervous System (ANS) changes during a mild sympathetic stimulation induced by a rest-to-stand protocol. The study will be conducted on a cohort of 60 healthy subjects.

The paper is organized as follows: Section 2 describes the experimental setup, including video and ECG recordings, the algorithms used to extract the vPPG signal and the HRV and PRV series. Performances of the methods and the comparison between PRV and HRV parameters on the population are described in Section 3. Finally, discussion is reported in Section 4.

\section{Material and Methods}

\subsection{Video and ECG recordings}

Video recordings were performed through a GigE Sony XCG-C30C camera, whose image sensor is $1 / 3$ " color progressive scan with spatial resolution of $659 \times 494$ pixels. Videos were acquired with a frame-rate of 60 frames-per-second (fps) and 8-bits resolutions. Data were saved in RGB, uncompressed, AVI raw format to be processed offline. The camera was equipped with $15 \mathrm{~mm}$ fixed focal length lenses (Tamron $25 \mathrm{HB}$ ), which were used to magnify the region occupied by the partecipant's face. The camera was fixed on a tripod, which was equipped with a mechanical arm to allow the manual adjustment of camera's positions when moving from one phase of the experiment to another.

The ECG signal was recorded by the FlexComp Infiniti by Thought Technologies, Inc. Electrodes were located on the body surface as follows: the negative electrode was placed on the right shoulder, the positive electrode on the left side of the chest and the ground electrode on the left shoulder. ECG traces were sampled at $256 \mathrm{~Hz}$. 


\subsection{Experimental Procedure}

A group of 60 healthy volunteers (31 males and 29 woman) with different ages (24 \pm 3 mean \pm SD, range $20-34$ ) were recruited for this study. Participants had various skin types ranging from type II to type IV.

All the recordings were conducted indoor, with a changing mixture of sunlight passing through the window and artificial light provided by illumination indoor. All the participants received a complete explanation of the above procedure and signed a comprehensive, written informed consent. Subjects underwent to a classical restto-stand maneuver, which is known to elicit sympathetic responses. During rest, the subjects were asked to lay on a bed facing the camera. The bed was positioned in front of the window to have direct sunlight on subject's face. Participants were instructed not to move their head in order to reduce movement artifacts. After a time period of 2-3 minutes, needed to relax the subjects and to stabilize their cardiac frequency, video and ECG recording were started and continued for 5 minutes. Next, the subjects were asked to stand up, while the camera was manually moved to follow their face. During this period both video and ECG recording were suspended. When subjects had reached a stable position and camera was properly aligned, ECG and video recordings were restarted for a period of five minutes.

\subsection{Extraction of $v P P G$ Signal}

Videos were processed offline to derive the vPPG signal. Three regions of interests (ROI) were considered: forehead $\left(R O I_{1}\right)$, nose $\left(R O I_{2}\right)$ and cheek $\left(R O I_{3}\right)$. ROI detection and tracking were developed in OpenCV: detections were performed by the Viola-Jones face detection algorithm [14] while ROI tracking was obatined by the Lucas-Kanade-Tomasi motion flow tracking algorithm (LKT) [15].

Let's indicate as $\mathrm{M}$ the number of color channels ( $M=3$, the $\mathrm{R}, \mathrm{G}$ and $\mathrm{B}$ channels), as $\mathrm{N}$ the number of ROIs ( $N=3$ namely forehead, nose and cheek ROIs) and as $\mathrm{L}$ the number of frames of video recording. In each ROI, a spatial average of pixels intensity was calculated for each frame, thus obtaining $M \times N$ time series of $L$. These time series were firstly detrended using the technique described in [16], setting the smoothness parameter $\lambda=400$. A hamming bandpass filter with cut-off frequencies of $f_{1}=0.1, f_{2}=8 \mathrm{~Hz}$ was then applied to remove the high frequencies components, mainly correlated to artificial light, and the low frequencies components, associated to slow subject movements.

After this preprocessing, the vPPG signal was extracted using three methods: i) the chrominance model ; ii) the Zero-phase Component Analysis (ZCA) method and iii) a method based on the combination of chrominance model and ICA (ChromICA). Those will be briefly described in the following:

2.3.1. Chrominance model. According to the deHaan's work [7], it is possible to maximize the pulsate component of the reflected light and minimize extraneous noise 
by an appropriate weighing of R, G and B components:

$$
S=c_{1} R+c_{2} G+c_{3} B
$$

where $c_{1}=3\left(1-\frac{\alpha}{2}\right), c_{2}=-2\left(1+\frac{\alpha}{2}\right), c_{3}=\frac{3 \alpha}{2}$ are the linear coefficients and $\alpha=\frac{\sigma(X)}{\sigma(Y)}$ is the normalization factor that takes into account different amplitudes of the two chrominance signals $X$ and $Y$.

2.3.2. $Z C A$ method. The $\mathrm{ZCA}$ method can be seen as a variant of Principal Component Analysis (PCA). Let's $\mathbf{X}$ an $L \times M$ matrix having the $\mathrm{R}, \mathrm{G}$ and $\mathrm{B}$ channels in its columns and the $L$ points in its rows. Let's also assume that the covariance $\mathbf{C}=\mathbf{X}^{T} \mathbf{X}$ has eigenvectors in the columns of $\mathbf{E}$ matrix and the eigenvalues on the diagonal of $\boldsymbol{\Sigma}$, so that $\mathbf{C}=\mathbf{E} \mathbf{\Sigma} \mathbf{E}^{T}$. We are interested to find a matrix $\mathbf{W}$ so that the transformation:

$$
\mathbf{Y}=\mathbf{W X}
$$

will produce a set of new variable $\mathbf{Y}$ which are uncorrelated and have unit variance (i.e. $\left.\mathbf{Y}^{T} \mathbf{Y}=\mathbf{I}\right)$. In the PCA approach, the whitening matrix is:

$$
\mathbf{W}_{P C A}=\boldsymbol{\Sigma}^{-1 / 2} \mathbf{E}^{T}
$$

This transformation first rotates the variables using the eigenvectors in the matrix $\mathbf{E}$. This results in orthogonal components characterized by different variances. To achieve whitened data, the rotated variables are scaled by the square root of the eigenvalues $\Sigma^{-1 / 2}$. Because of the rotation, the decorrelated channels will be shuffled respected to the order of the input data. However, since the pulse information is mainly contained in the $\mathrm{G}$ channel (as shown by Verkruysse et al. [17]), a method that could avoid the shuffle of the channels would facilitate the selection of the channel containing the main videoPPG information. One possible solution is represented by zero phase component analysis (ZCA). In ZCA the following transformation matrix is used:

$$
\mathbf{W}_{Z C A}=\mathbf{E} \Sigma^{-1 / 2} \mathbf{E}^{T}
$$

In this case the second rotation given by $\mathbf{E}$ rotates back the data to the original coordinate system. Therefore the term zero phase indicates the forward-backward projection of the data to the original space.

The ZCA is also called Mahalanobis transformation and, according to [18], among the whitening methods, it is the unique method that, by maximizing the cross-covariance $\Phi$ between the original data $\mathbf{X}$ and whitened data $\mathbf{Y}$, can minimize the total squared of their distance (defined as Mahalanobis distance):

$$
\mathbf{d}(\mathbf{X}, \mathbf{Y})=\sqrt{(\mathbf{X}-\mathbf{Y})^{\mathbf{T}} \boldsymbol{\Phi}^{-\mathbf{1}}(\mathbf{X}-\mathbf{Y})}
$$

2.3.3. ChromICA method. Among the population a bulk of subjects moved excessively their heads during stand position. In this cases the Chrom method lacked of robustness to recover the pulse cardiac information. We therefore decided to combine the Chrom method useful to exploit the BVP signal, and ICA to separate better the motion artifact 
to the desired signal.

For each frame the ROI tracking gives the position $x-y$ of each region. After detrending and band-pass filtering the time series, two signals associated to the ROIs movement is available. Together with the chrominance signal a matrix of $3 \times N_{\text {data }}$ was created for each ROI and ICA decomposition, based on joint approximate diagonalization of eigenmatrices (JADE) algorithm [19] was applied to separate the motion artifact to the pulse signal. However since ICA decomposition does not assure the order of otuput channels, the ZCA decorrelation has been implemented in the JADE algorithm.

Each method was applied on a window of 5 seconds to account to nonstationarity noise as motion artifact and abrupt changes in reflecting light. Within each epoch, a coarse estimate of pulse rate $f_{c}$ was obtained by the power spectral density (PSD) of the windowed signal. The estimate of pulse rate can be erroneous at time, so a track of the history of pulse rates over the past epochs was accomplished. If the current $f_{c}$ is off by more than $20 \%$, its value is substituted with the median of previous pulse rates estimates.

We therefore obtained three vPPG signals, one from each of the analysed ROI. Among them, the selection of the target vPPG signal was achieved by calculating the power spectral density (PSD) on the entire signal and by measuring the $S N R$ using the following formula:

$$
S N R=10 * \log _{10}\left(\frac{\int_{f_{1}}^{f_{2}} \mathcal{P}_{v P P G_{i}}(f) \mathrm{d} f}{\int_{0.1}^{f_{1}} \mathcal{P}_{v P P G_{i}}(f) \mathrm{d} f+\int_{f_{2}}^{4} \mathcal{P}_{v P P G_{i}}(f) \mathrm{d} f}\right)
$$

where $\left(\mathcal{P}_{v P P G_{i}}(f)\right.$ with $\left.i \in\left\{1,2, \ldots, N_{R O I}\right\}\right)$ is the PSD of $i^{\text {th }}$ vPPG signals, $f_{1}=$ $f_{p}-0.15, f_{2}=f_{p}+0.15$ and where $f_{p}$ is the pulse frequency (measured in Hz). The signal with the highest SNR was selected as target vPPG signal. Finally, vPPG signal was interpolated using cubic splines and resampled at $256 \mathrm{~Hz}$, to match the temporal resolution of the ECG signal.

\subsection{Extraction of $R R$ and $P P$ series}

The detection of pulse waves $(\mathrm{P})$ of $\mathrm{vPPG}$ was performed on the first derivative of the signal by applying the algorithm proposed by Scholkmann et al [16]. Since the detection of the peak was done on the derivative of vPPG signal, the position of each pulse will be located on the maximum of first derivative, i.e. along the rising edge of the cardiac pulse (defined as anachrotic phase). The PP series were extracted as the series of time difference between two consecutive $\mathrm{P}$ positions. To correct possible miss-detected pulses, the adaptive filtering algorithm proposed by Wessel et al [20] was applied on PP series.

The detection of $\mathrm{R}$ peaks in the ECG was automatically performed by applying the method of Alfonso et al [21]. The $\mathrm{R}$ detections were then visually inspected and the misdetected/erroneous beats were corrected using an interactive home-made software. Finally, the RR interval series were extracted as the series of time difference between 
two consecutive $\mathrm{R}$ positions. The correctness of $\mathrm{R}$ detections and $\mathrm{RR}$ series is needed because they will represent the gold standard for the comparison of vPPG methods.

\subsection{Quantification of HRV parameters}

Analysis of HRV was performed in both time and frequency domain using classical timeinvariant, linear methods as described in [22]. In time-domain, we computed the mean of normal-to-normal intervals (NN), the standard deviation of all NN intervals (SDNN), the square root of the mean of the sum of the squares of differences of adjacent NN (RMSSD) and the proportion of NNs that differ by more than $50 \mathrm{~ms}$.

In frequency domain, the corrected $\mathrm{PP}$ and $\mathrm{RR}$ series were resampled at $2 \mathrm{~Hz}$ and the PSD of Heart Rate Variability (HRV) and Pulse Rate Variability (PRV) was computed by Welch power spectral densitiy estimate in order to analyze the parasympathetic and sympathetic activity of ANS . According to [22], the spectrum of these signals is characterized by three main spectral components: the very low frequency (VLF) component in the frequency band $[0.003-0.04] \mathrm{Hz}$, the low frequency (LF) component in the $[0.04-0.15] \mathrm{Hz}$ band and the high frequency (HF) component in the $[0.15-0.4] \mathrm{Hz}$ band. Although there is controversy on the physiological meaning of each component, it is generally assumed that the LF corresponds to sympathetic and parasympathetic modulation, while the $\mathrm{HF}$ is associated to parasympathetic modulation. Finally, the ratio between LF and HF measures the sympatho/vagal balance responsible of heart rate modulation. The quantitative evaluation of the power associated to the LF, HF components have been conducted by determining the area under the PSD of RR/PP intervals. In this study, the LF and HF components are presented in normalized units.

\section{Results}

Two subjects out of 60 were excluded from the analysis: in details, one subject was moving excessively during stand session, while for the second one the stand session was not acquired for technical problems. We therefore present the results of 58 subjects. Results will be divided in two sections: one is dedicated to the comparison of methods performance and the second regards the comparison between HRV and PRV parameters.

\subsection{Performances of the methods}

Fig. 1 shows the average SNR (computed by equation (6)) of vPPG signal extracted with the three considered methods (Chrom, ZCA, ChromICA) in the ROIs of interest. During rest (Fig. 1a), the averaged SNR is almost equivalent for Chrom and ZCA methods, while it is slightly worse when ChromICA is used, especially for $R O I_{1}$ (forehead). During stand (Fig. 1b), the SNR is lower than in rest condition thus evidencing a more problematic extraction of vPPG signal in stand conditions. Comparing the methods, 
Table 1: Mean beat detection accuracy according to $S e,+P$ and $A c c$ over all subjects for rest and stand conditions.

\begin{tabular}{lccc}
\hline & $\begin{array}{c}\text { Sensitivity } \\
\text { (all values are expressed in mean } \pm \text { std) }\end{array}$ & Accuracy \\
\hline At rest & & & \\
Chrom & $0.989 \pm 0.010$ & $0.988 \pm 0.029$ & $0.979 \pm 0.036$ \\
ZCA & $0.996 \pm 0.006$ & $0.992 \pm 0.015$ & $0.988 \pm 0.019$ \\
chromICA & $0.988 \pm 0.010$ & $0.972 \pm 0.026$ & $0.961 \pm 0.032$ \\
At stand & & & \\
Chrom & $0.969 \pm 0.080$ & $0.941 \pm 0.175$ & $0.927 \pm 0.180$ \\
ZCA & $0.986 \pm 0.039$ & $0.974 \pm 0.083$ & $0.961 \pm 0.093$ \\
chromICA & $0.966 \pm 0.079$ & $0.928 \pm 0.143$ & $0.911 \pm 0.149$ \\
\hline
\end{tabular}

the ZCA method has a slightly better performance in all the three ROIs, while the ChromICA has always the worse performance.

(a)

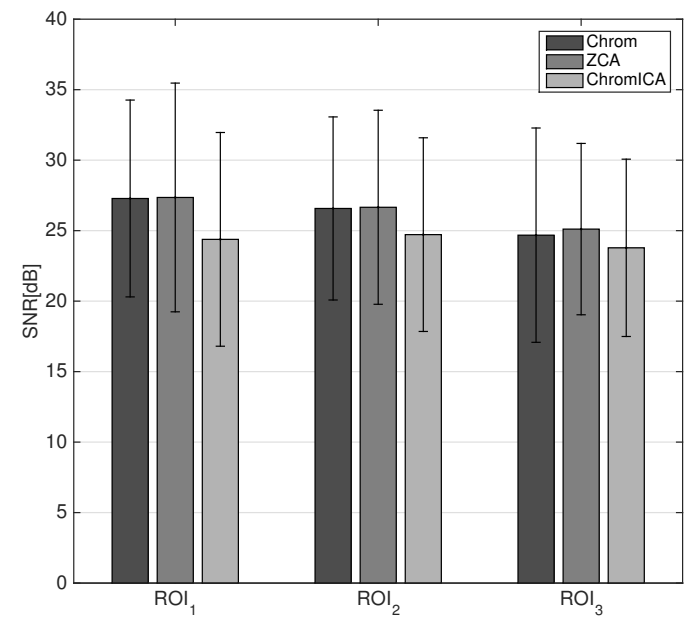

(b)

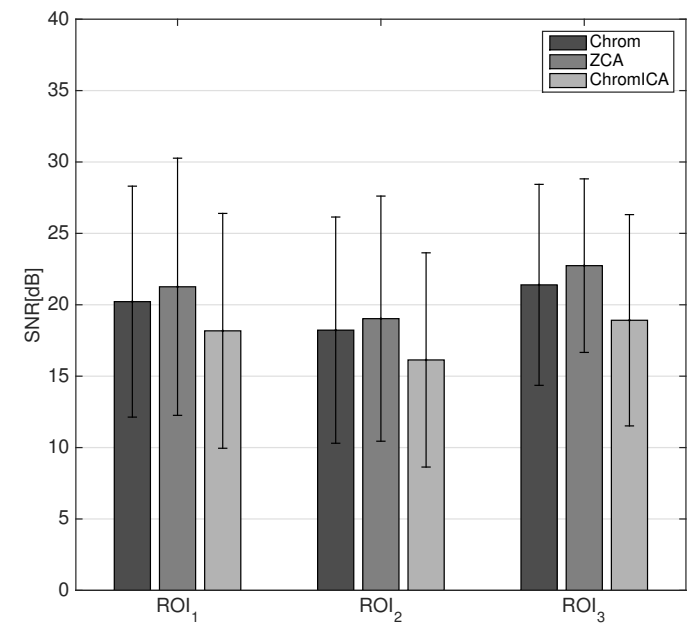

Figure 1: Comparison of SNR obtained for all subjects and different facial regions $\left(R O I_{1}, R O I_{2}\right.$, $\mathrm{ROI}_{3}$ ). (a) Rest session; (b) Stand session.

Pulse detection performances are reported in Tab. 1 in terms of sensitivity, positive prediction and accuracy. Very excellent performances are observable during rest, but all the methods share a performance drop passing from rest to stand session. However, the lowest reduction of performances is experienced when vPPG is obtained using ZCA method.

We finally compared the methods in terms of computational load (calculated in the proprietary commercial software Matlab running on Macbook Air, Processor Intel i5), measured as the computation time needed to process the RGB signals and to extract the vPPG signal. Tab. 2 reports the averaged computational load for all the methods. As the Chrom method is based on a a priori model and only needs the calculation of 
Table 2: Mean computational load calculated for the three different methods Chrom, ZCA and ChromICA needed to process 5 sec windowed signal.

\begin{tabular}{lr}
\hline $\begin{array}{l}\text { Method } \\
\text { (expressed in ms) }\end{array}$ \\
\hline Chrom & $2.78 \pm 0.46$ \\
ZCA & $3.21 \pm 0.76$ \\
ChromICA & $5.42 \pm 1.54$ \\
\hline
\end{tabular}

Table 3: Comparison of temporal and frequency parameters between the ECG sensor and the video camera considering rest-to-stand session $\left({ }^{*}\right.$ pvalue $\left.<0.05\right),{ }^{* *}$ pvalue $\left.<0.01\right)$.

\begin{tabular}{lcccc}
\hline & \multicolumn{2}{c}{ ECG } & \multicolumn{2}{c}{ vPPG } \\
\hline & Rest & Stand & Rest & Stand \\
HR(Hz) & $1.15 \pm 0.18$ & $1.37 \pm 0.20^{* *}$ & $1.15 \pm 0.18$ & $1,37 \pm 0,21^{* *}$ \\
SDNN(ms) & $52 \pm 24$ & $56 \pm 19$ & $50 \pm 17$ & $54 \pm 17$ \\
RMSSD(ms) & $24 \pm 8$ & $18 \pm 7^{* *}$ & $24 \pm 8$ & $24 \pm 10$ \\
pNN50(\%) & $6 \pm 6$ & $3 \pm 4^{* *}$ & $6 \pm 6$ & $6 \pm 7$ \\
LF(\%) & $28 \pm 14$ & $45 \pm 16^{* *}$ & $26 \pm 12$ & $30 \pm 13^{*}$ \\
HF(\%) & $64 \pm 13 \%$ & $47 \pm 14^{* *}$ & $61 \pm 11$ & $50 \pm 10^{* *}$ \\
LF $/ H F$ & $0.51 \pm 0.38$ & $1.17 \pm 0.79^{* *}$ & $0.49 \pm 0.34$ & $0.70 \pm 0.51^{* *}$ \\
\hline
\end{tabular}

the coefficients $c_{1}, c_{2}, c_{3}$ of equation (1), this method is faster than ChromICA and ZCA, which have to to separate the sources by heuristic approach.

According to the above results, ZCA method showed to be a good compromise between robustness and speed. Therefore $\mathrm{vPPG}$ signal extracted by this method was selected for the successive variability analysis.

\subsection{Comparison between $P R V$ and HRV parameters}

In Fig. 2 the correlations calculated over all the population between ECG and vPPG regarding temporal parameters are reported. During rest session the Pearson correlation remains high $(r>0.90)$ for the whole parameters. In stand session the mean heart rate and the SDNN maintained high correlation, while RMSSD and pNN50 values are distributed in a wider cloud, therefore the correlation falls. A confirmation of worse RMSSD results during stand session is shown in the Tab. 3, where the RMSSD estimated on PP series in stand session is higher both in mean and standard deviation compared to the RMSSD estimated on RR series.

In Fig. 3 the correlations over all the population between ECG and $\mathrm{vPPG}$ of spectral parameters LF, HF and LF/HF are reported. During the rest session there is 

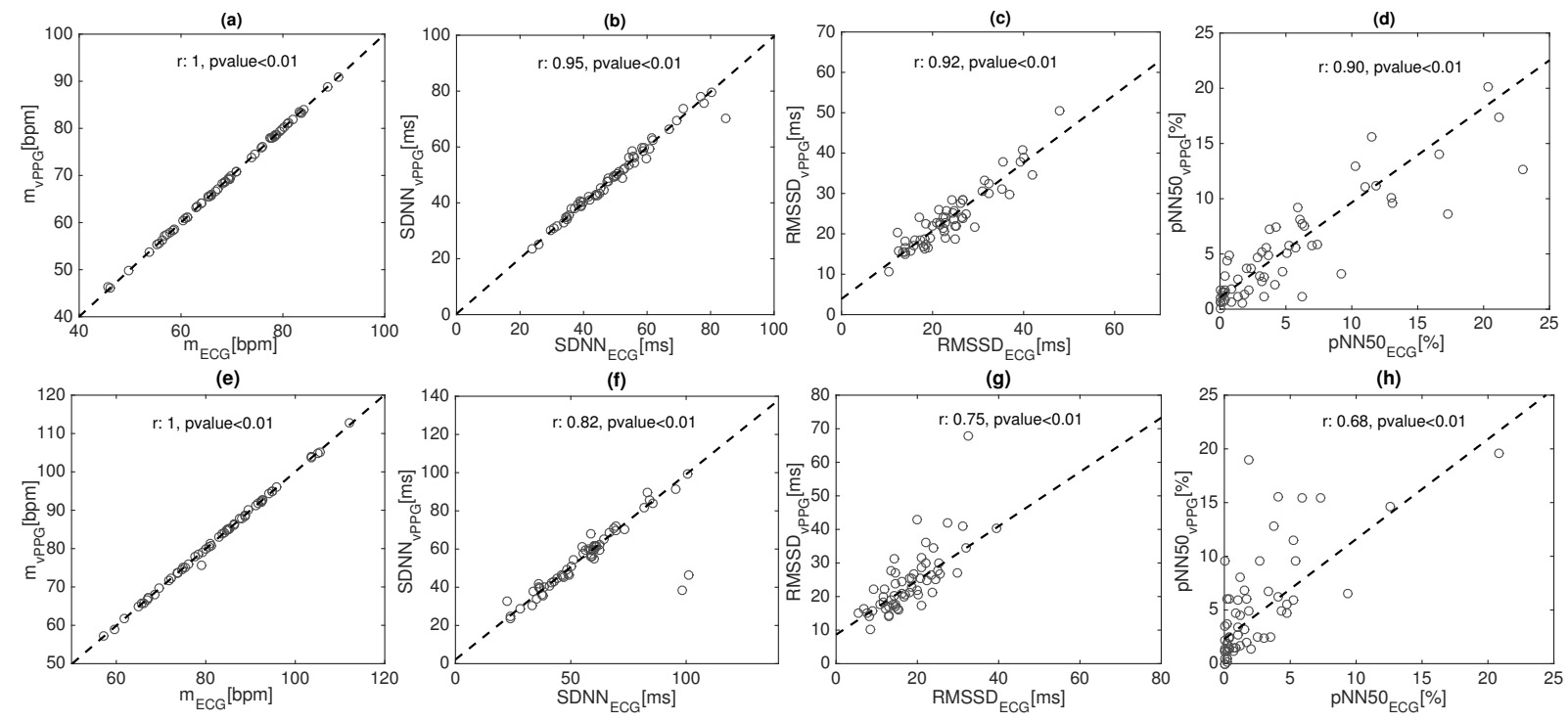

Figure 2: Scatter plots comparing the temporal measurements of (a-e) heart rate (HR), (b-f) SDNN, (c-g) RMSSD, (d-h) pNN50, for the ECG and vPPG signal. Upper row: Rest session; Lower row: Stand session.

a great correspondence between PRV and HRV spectral parameters $(r>0.9)$. However in stand position there is an evident drop of correlations in $\mathrm{LF}, \mathrm{HF}$ and $\mathrm{LF} / \mathrm{HF}$. In Tab. 3 it is possible to notice that during stand session there is a negative bias of the mean of LF and an overestimation of the mean of HF component estimated from PP series compared to LF and HF calculated from RR series. Therefore the sympato-vagal balance expressed by the $\mathrm{LF} / \mathrm{HF}$ ratio is clearly underestimated, and a bias of 0.5 is expressed between the vPPG system and the ECG. Although the sympato-vagal balance value obtained by ${ }^{2} P P G$ is underestimated, Tab. 3 shows that there is a statistically difference in the $\mathrm{LF} / \mathrm{HF}$ between rest and stand sessions, detectable by both on HRV and PRV.

\section{Discussions}

A few scientific articles have investigated the PRV obtained by vPPG signal $[3,4,6]$. However none of them have tested how vPPG-derived autonomic indexes are modified during autonomic stimulation and have validated the vPPG-derived PRV as a good surrogate of HRV.

The extraction PRV parameters from vPPG is not novel. Poh et al. [3] were the first that implemented a fully automated method to extract from vPPG signal the spectral parameters of PRV as LF, $\mathrm{HF}$ and $\mathrm{LF} / \mathrm{HF}$ ratio, even if the time recording was quite short (1 minute only) for an effective computation of spectral parameters [22]; in addition, the analysis was performed on rest state, only.

McDuff et al. [6] improved the previous work studying the sympathetic activation during a session of rest/stress task. The results in terms of spectral parameters (LF,HF 

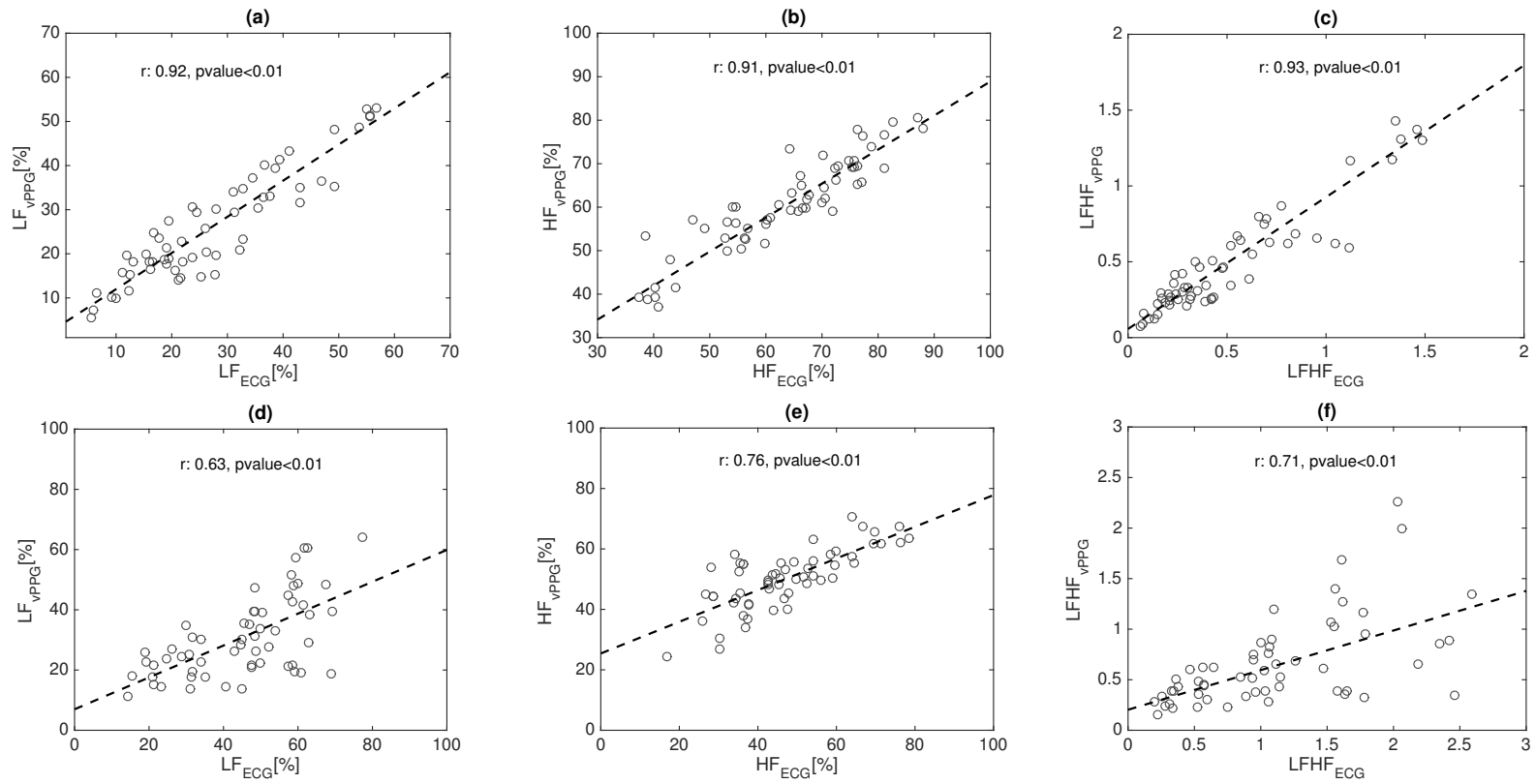

Figure 3: Scatter plots comparing the spectral measurements of (a-d) LF, (b-e) HF, (c-f) $\mathrm{LF} / \mathrm{HF}$ ratio, for the ECG and $\mathrm{vPPG}$ signal. Upper row: Rest session; Lower row: Stand session.

and $\mathrm{LF} / \mathrm{HF}$ ratio) showed a good correlation $(r>0.86)$ between the vPPG system and the contact PPG sensor. Although the article demonstrated the feasibility of $v \mathrm{PPG}$ technology to monitor stress condition, some limitations arised: the PRV analysis was limited to frequency domain only; the use of ICA method implied the implementation of heuristic approach for the selection of the output channel that can fail when strong periodic subject movements are present.

Similar comparisons between PRV and video-PRV have been performed in other works $[4,10]$, but this comparison is not exhaustive as it is know that PRV and HRV may differ [23] especially during sympathetic stimulations. Only one previous work [11] studied the temporal and spectral analysis comparing the HRV obtained by ECG chest strap and the PRV obtained by the vPPG system during supine and sitting sessions. However, the subjects acquired in supine position were different from those recorded in sitting position and thus no evaluation of the capability of the PRV to describe autonomic changes in the same subject was achieved. Moreover no automatic method was developed to identify the ROI (done manually) and a controlled illumination of the environment was performed in order to reduce artificial light interference.

To the extent of our knowledge, this work is the only one which investigate the vPPG derived parameters as a surrogate of HRV. Our results show that during rest session both short-term (RMSSD, pNN50, HF) and long-term variability parameters $(\mathrm{LF}, \mathrm{SD})$ are in good agreement (correlation $r>0.91$ ). However during stand session the concordance deteriorated especially in all short-term variability parameters and $\mathrm{LF} / \mathrm{HF}$ ratio exhibits positive bias. This deterioration does not affect the capability to 
detect autonomic changes in LF and HF components passing from rest-to-stand.

Temporal parameters pNN50 and RMSSD evidenced the largest differences: if pNN50 and RMSSD calculated in ECG are significant different passing from rest to stand, the same is not observable in vPPG. The difference in pNN50 and RMSSD can be explained by the lower sampling frequency of video recording $(60 \mathrm{~Hz})$, which generates a smooth vPPG signal and induces jitter in vPPG fiducial points detection in the range of $( \pm 1 / 60 \approx \pm 17)$. This certainly affects PRV interval measurements.

Even if this problem can be overcome in future work by using higher video sampling rate, it is important to observe these disagreements are also detected using contact PPG recordings as reported in the review by Schäfer et al. [23] on the accuracy of PRV as an estimate of HRV. Studies conducted on rest-to-stand or rest-to-tilt protocol [24-26], showed an increased bias during sympathetic stimulus, especially in short-term variability parameters, like RMSSD, pNN50. This difference can be explained by PulseTransit Time (PTT) variability [27], which highly modulates high-frequency components of PRV. PTT influences seem to be predominant during stand.

From a methodological point of view, we have investigated a new method for the extraction of $\mathrm{vPPG}$ signal on short time segment ( 5 seconds) with potential for real-time acquisitions. The method is based on ZCA, a variant of PCA. The advantage of using ZCA method is twofold: firstly, ZCA, by projecting data back to the original space, avoids the searching of pulse signal among the detected components (required for PCA or ICA approaches); secondly ZCA is not affected by flipping (i.e. inversion of the sign from one segment to the consecutive ones) as it may happen in ICA. Our approach has been proved to be robust in comparison with Chrom and ChromICA. We observed that vPPG reconstructed by ZCA has a slightly better SNR than those reconstructed by Chrom method, especially in the most critical situation (i.e. during stand). In addition,

the location of pulse waves was superior in terms of both sensitivity and specificity using our approach. Conversely, Chrom method was the best one in term of computational burden.

The overall conclusion is that vPPG variability parameters can be considered as a good surrogate of HRV parameters during supine position, while more caution should be paid during stand, where the agreement deteriorates due to both to methodological (increased motion noises) and physiological reasons (i.e PTT). Nevertheless, we showed that the PRV extracted by vPPG is able to highlight the mild autonomic activation induced by stand and thus PRV parameters derived from vPPG can be used to assess autonomic responses.

\section{Bibliography}

[1] W. Verkruysse, L. O. Svaasand, and J. S. Nelson. Remote plethysmographic imaging using ambient light. Opt. Express, 16:21434-21445, 2008.

[2] C. Takano and Y. Ohta. Heart rate measurement based on a time-lapse image. Med. Eng. Phys., 29:853-857, 2007. 
[3] M.-Z. Poh, D. McDuff, and R. W. Picard. Advancements in noncontact, multiparameter physiological measurements using a webcam. IEEE Trans. On Biomed. Eng., 58:7-11, 2011.

[4] Y. Sun, S. Hu, V. Azorin-Peris, R. Kalawsky, and S. Greenwald. Noncontact imaging photoplethysmography to effectively access pulse rate variability. J. of Biomed. Opt., 18:12051213, 2013.

[5] S. Zaunseder, A. Heinke, A. Trumpp, and H. Malberg. Heart beat detection and analysis from videos. IEEE Int. Sc. Conf. El. and Nan., 24:286-290, 2014.

[6] D. McDuff, S. Gontarek, and R. W. Picard. Improvements in remote cardiopulmonary measurement using a five band digital camera. IEEE Trans. on Biomed. Eng., 61:2593-2601, 2014.

[7] G. de Haan and V. Jeanne. Robust pulse rate from chrominance-based rppg. IEEE Trans. On Biomed. Eng., 60:2878-2886, 2013.

[8] M. Lewandowska, J. Rumiński, T. Kocejo, and J. Nowak. Measuring pulse rate with a webcam a noncontact method for evaluating cardiac activity. Proc. of the Fed. Conf. on Comp. Sc. and Inf. Syst., 22:405-410, 2011.

[9] E. B. Blackford and J. R. Estepp. Effects of frame rate and image resolution on pulse rate measured using multiple camera imaging photoplethysmography. Proc. of SPIE, 947:94172D1-94172D14, 2015.

[10] Freédéric Bousefsaf, Choubeila Maaoui, and Alain Pruski. Remote detection of mental workload changes using cardiac parameters assessed with a low-cost webcam. Computers in Biology and Medicine, 53:154-163, 2014.

[11] J. Moreno, J. Ramos-Castro2, J. Movellan, E. Parrado, G. Rodas, and L. Capdevila. Facial video-based photoplethysmography to detect HRV at rest. Sports Med., 36:1-7, 2015.

[12] L. Tarassenko, M. Villarroel, A. Guazzi, J .Jorge, D. A. Clifton, and C. Pugh. Non-contact videobased vital sign monitoring using ambient light and auto-regressive models. Institute of Physics and Engineering in Medicine., 35:807-831, 2014.

[13] D. McDuff, S. Gontarek, and R. W. Picard. Remote detection of photoplethysmographic systolic and diastolic peaks using a digital camera. IEEE Trans. on Biomed. Eng., 61:2948-2954, 2014.

[14] P. Viola and M. Jones. Rapid object detection using a boosted cascade of simple features. Proceedings of IEEE Conference on Computer Vision and Pattern Recognition, page 511, 2001.

[15] B. D. Lucas and T. Kanade. Detection and tracking of point features. Technical Report MU-CS91-132, Carnegie Mellon University, 1991.

[16] M. P. Tarvainen, P. O. Ranta-Aho, and P. A. Karjalainen. An advanced detrending method with application to HRV analysis. IEEE Trans. Biomed. Eng., 49:172-175, 2002.

[17] W. Verkruysse, L. O Svaasand, and J S. Nelson. Remote plethysmographic imaging using ambient light. Opt. Exp., 16:21434-21445, 2008.

[18] A. Kessy, A. Lewin, and K. Strimmer. Optimal whitening and decorrelation. stat.ME, pages 1-14, 2015.

[19] J. F. Cardoso. High-order contrasts for independent component analysis. Neural Computing, 11:157-192, 1999.

[20] N. Wessel, A. Voss, H. Malberg, Ch. Ziehmann, H.U. Voss, A. Schirdewan, U. Meyerfeldt, and J. Kurths. Nonlinear analysis of complex phenomena in cardiological data. Herzschr. Elektrophys., 11:159-173, 2000.

[21] V. X. Afonso, W. J. Tompkins, T. Q. Nguyen, and S. Luo. ECG beat detection using filter banks. IEEE Trans. Biomed. Eng., 46:192-202, 1999.

[22] Task Force of the European Society of Cardiology, the North American Society of Pacing, and Electrophysiology. Heart rate variability. Eur. Heart J., 17:354-381, 1996.

[23] A. Schäfer and J. Vagedes. How accurate is pulse rate variability as an estimate of heart rate variability? a review on studies comparing photoplethysmographic technology with an electrocardiogram. International Journal of Cardiology, 166:15-29, 2013.

[24] S. Carrasco, R. Gonzalez, J. Jimenez, and R. Roman V. Medina J. Azpiroz. Comparison of the 
heart rate variability parameters obtained from the electrocardiogram and the blood pressure wave. J. Med. Eng. Technol., 22:195-205, 1998.

[25] S. Lu, H. Zhao, K. Ju, K. Shin, M. Lee, K. Shelley, and K.H. Chon. Can photoplethysmography variability serve as an alternative approach to obtain heart rate variability information? J Clin. Monit. Comput., 22:23-29, 2008.

[26] K. Charlot, J. Cornolo, J. V. Brugniaux, J. P. Richalet, and A. Pichon. Interchangeability between heart rate and photoplethysmography variabilities during sympathetic stimulations. Physiol. Meas., 30:1357-69, 2009.

[27] E. Gil, M. Orini, R. Bailón, J.M. Vergara, L. T. Mainardi, and P. Laguna. Photoplethysmography pulse rate variability as a surrogate measurement of heart rate variability during non-stationary conditions. Physiol. Meas., 31:1271-90, 2010. 